The Free Internet Journal

for Organic Chemistry
Paper

Arkivoc 2017, part iv, 210-221

\title{
Synthesis of novel benzimidazole-diindolylmethane hybrid compounds within the green chemistry context
}

José Guillermo Penieres-Carrillo, ${ }^{a *}$ Ricardo Alfredo Luna-Mora, ${ }^{a, b}$ José Guadalupe López-Cortés, ${ }^{b}$ Fernando

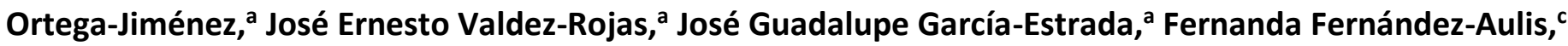
and Cecilio Álvarez-Toledano ${ }^{b}$

a Sección de Química Orgánica, Facultad de Estudios Superiores Cuautitlán, Universidad Nacional Autónoma de México, Campo 1, Avenida 1 de mayo s/n, Colonia Santa María Guadalupe Las Torres, Cuautitlán Izcalli, Estado de México, C.P. 54740, México

${ }^{b}$ Instituto de Química, Universidad Nacional Autónoma de México, Ciudad Universitaria, Circuito Exterior, México D.F., C.P. 04510, México

' Departamento de Alimentos y Biotecnología, Facultad de Química, Edificio E, UNAM, Av. Universidad 3000, C.U., Coyoacán, México, D.F., 04510, México

E-mail:penieres@unam.mx

Received 11-29-2016

Accepted 04-17-2017

Published on line 05-26-2017

\section{Abstract}

The syntheses of novel hybrid 2-(3,3'-diindolylmethylphenyl)-1H-benzimidazole regioisomers under ecofriendly, solvent-less, catalyst-free conditions and using microwave energy with good to excellent yields in short reaction times were achieved.
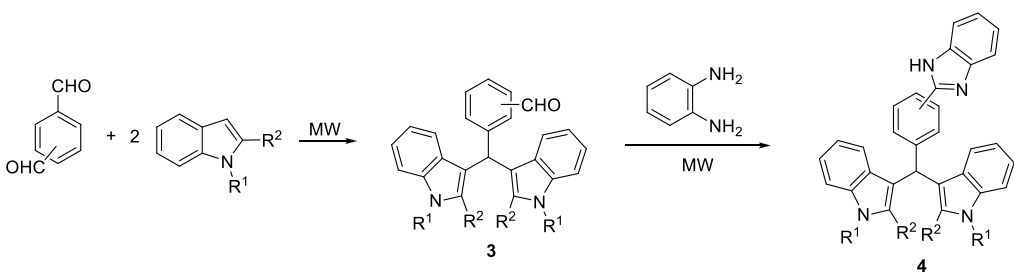

Keywords: Benzimidazoles, diindolylmethanes, aryldialdehydes, o-phenylenediamine, microwave energy 


\section{Introduction}

3,3'-Diindolylmethane (DIM, Figure 1A), the major acid condensation product of indole-3-carbinol (I3C, Figure 1B), is a promising antitumor agent derived from Brassica (Cruciferous) vegetables. ${ }^{1}$ The anticarcinogenic effects of DIM have been shown in animal models of spontaneous, carcinogen-induced or transplanted tumors. ${ }^{2,3}$ Because of their effectiveness and low toxicity, I3C and DIM have become widely used in adjunct therapies for recurrent respiratory papillomatosis (RRP), caused by some types of human papilloma viruses (HPVs). ${ }^{4,5}$

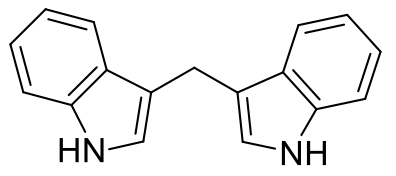

$1 \mathrm{~A}$

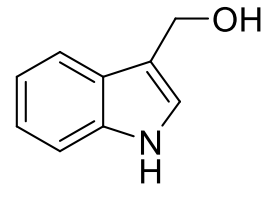

$1 B$

Figure 1. Structures of DIM (1A) and I3C (1B).

The benzimidazole pharmacophore is known to be an important structural core in medicinal chemistry that shows a broad spectrum of pharmacological activities. Several compounds containing the benzimidazole scaffold have been used as antiparasitic, ${ }^{6}$ antimicrobial, ${ }^{7}$ antitumor, ${ }^{8}$ and antihistaminic agents. ${ }^{9}$

The main methodology to synthesize DIM and its derivatives is by a condensation reaction between an indole with either aliphatic or aromatic aldehydes or ketones employing Broensted-Lowry acids ${ }^{10}$ or Lewis acids. ${ }^{11}$ Some of these reactions have long reaction times ${ }^{12}$ or low yields of products. ${ }^{13}$ Benzimidazole derivatives have usually been synthesized by classical cyclocondensation of $o$-phenylenediamines with the corresponding carboxylic acids under harsh dehydrating reaction conditions ${ }^{14}$ or from aldehydes under oxidative conditions. Some reagents such as nitrobenzene ${ }^{15}$ and sodium metabisulfite, ${ }^{16,17}$ have been employed for this last purpose.

On the other hand, microwave irradiation is well known to promote the syntheses of a great variety of compounds, ${ }^{18-20}$ where chemical reactions are accelerated because of selective absorption of microwaves by polar molecules and the coupling of these two factors under solvent-free conditions has received notable attention. ${ }^{21} \mathrm{~A}$ literature survey reveals examples of specific reactions, which do not occur under conventional heating, but could be possible by microwave irradiation. ${ }^{22}$

The synthesis of some DIM derivatives using catalysts such as silica sulfuric acid ${ }^{23}$ under thermal conditions involved reactions that took a long time and provided low to moderate yields. In some cases, many byproducts were formed when the reactions were carried out in aqueous medium and under controlled conditions at $\mathrm{pH}$ values of 1.0, 1.5, 2.5 and 7.2, respectively. ${ }^{24}$ In recent years, diindolylmethane synthesis has taken a greener direction, such as: using infrared energy in solvent-less conditions and in presence of a bentonitic clay, ${ }^{25}$ with ultrasound energy and aminosulfonic acid as catalyst, ${ }^{26}$ under microwave solvent-free irradiation with Lewis acid-catalysis, ${ }^{27}$ in ionic liquids, ${ }^{28-30}$ with SBA-15-supported poly[4styrenesulfonyl(perfluorobutylsulfonyl)imide] as heterogeneous Broensted-Lowry acid catalyst, ${ }^{31}$ using Montmorillonite K-10 clay, $^{32,33}$ with nickel nanoparticles as a reusable catalyst under solvent-free conditions, ${ }^{34}$ employing ion exchange resins, ${ }^{35,36}$ with eutectic salts, ${ }^{37}$ synthesis mediated by Zeokarb-225, ${ }^{38}$ and with zeolites, $^{14,39}$ amongst others. 
As a continuation of our interest in the syntheses of diindolylmethane derivatives, herein we report the synthesis of novel hybrid benzimidazole-diindolylmethane compounds using some principles of green chemistry such as the use of microwave energy source for the activation of reactions under solvent-free and catalyst-free conditions.

\section{Results and Discussion}

Following two previously established possible routes by us to synthesize 2-(4-(bis(1H-indol-3yl)methyl)phenyl)-1H-benzimidazole, we started with o-phenylenediamine and terephthalaldehyde to obtain 1 (Scheme 1), in order to generate compound 4a by microwave irradiation but the main obtained product was 2 in $80 \%$ yield.

A second synthetic route, which used $1 H$-indole and terephthalaldehyde, was successful and we obtained the diindolylmethane $\mathbf{3 a}$ in $96 \%$ yield in $8 \mathrm{~min}$ of reaction time by microwave irradiation (Scheme 2). Then, a mixture of $o$-phenylenediamine and 1 eq of 3 a were irradiated with microwave energy for 3 min and the product 4 a was generated in $88 \%$ reaction yield.

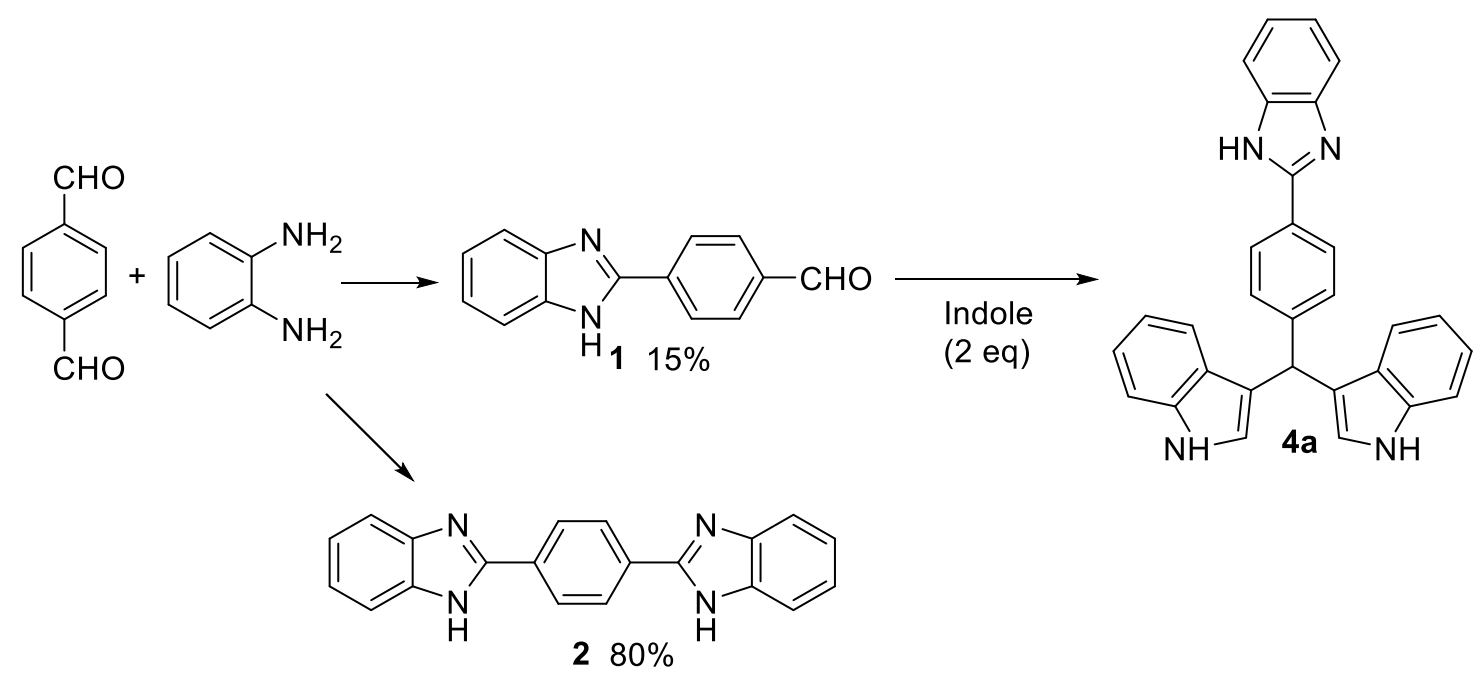

Scheme 1. Synthetic route $A$ for $4 a$.

Encouraged by this result, we realized the synthesis of $\mathbf{4 a - 4 1}$ compounds, Table 1 , using different indole derivatives and the 3 regioisomers of formylbenzaldehyde, according with the Scheme 3.

Compounds $\mathbf{3 a}$ and $\mathbf{3} \mathbf{b}$ had been previously reported, ${ }^{40-42}$ apparently as byproducts when trying to synthesize the corresponding 1,4-bis[bis(2-aryl-1H-indol-3-yl)methyl]benzene derivatives. According to our knowledge, the synthesis of all other (formylphenyl)Idiindolylmethane and (diindolylmethyl)phenylbenzimidazole derivatives presented here have not been reported, hence another point in the novelty of this work. 

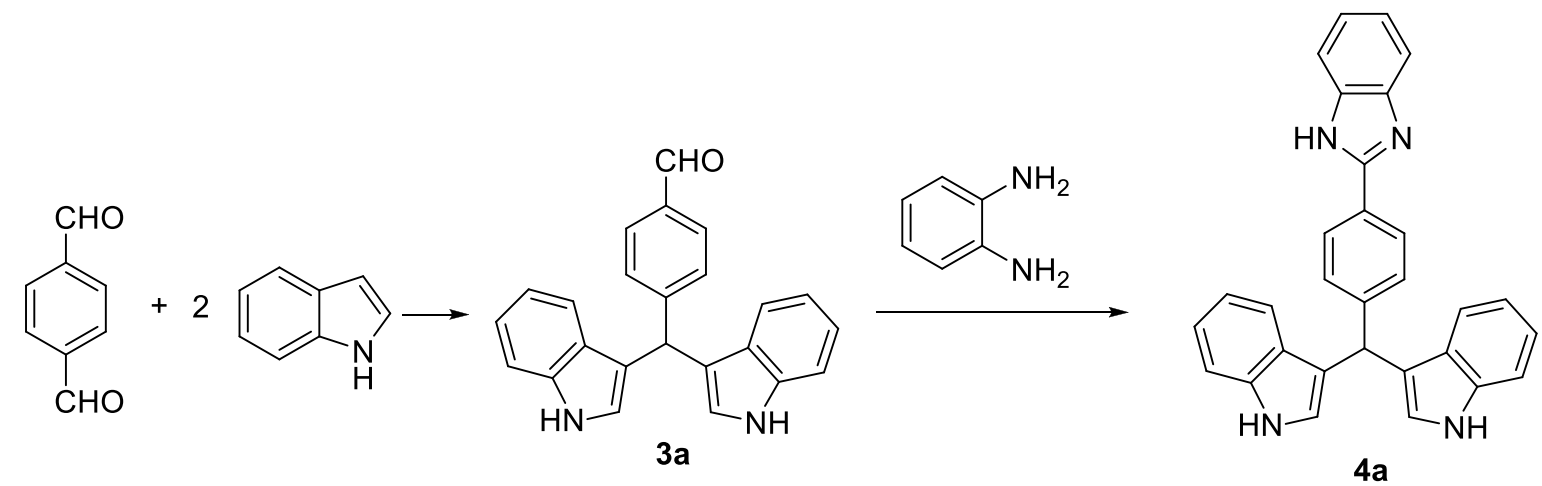

Scheme 2. Synthetic route B for $4 a$.

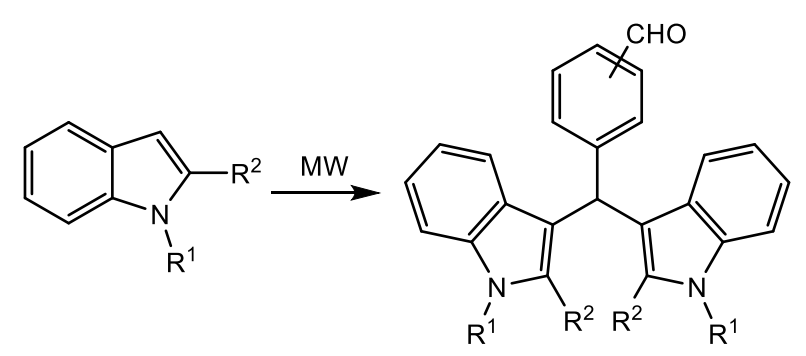

3

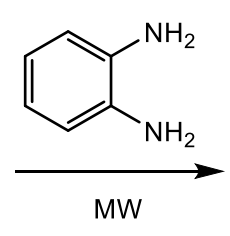<smiles>[R]c1[nH]c2ccccc2c1C(c1ccccc1)c1c([R])n([R1])c2ccccc12</smiles>

4

Scheme 3. Synthesis of compounds 3 and 4.

Table 1. Synthesis of compounds $\mathbf{3}$ and $\mathbf{4}$ by microwave irradiation

\begin{tabular}{|c|c|c|c|c|}
\hline Compound & Indole & Aldehyde & $\% 3^{a, b}$ & $\% 4^{\mathrm{c}, \mathrm{b}}$ \\
\hline a & $R^{1}=H \quad R^{2}=H$ & terephthalaldehyde & 96 & 88 \\
\hline b & $\mathrm{R}^{1}=\mathrm{Me} \mathrm{R}^{2}=\mathrm{H}$ & terephthalaldehyde & 93 & 92 \\
\hline c & $R^{1}=H \quad R^{2}=M e$ & terephthalaldehyde & 94 & 94 \\
\hline d & $\mathrm{R}^{1}=\mathrm{H} \mathrm{R}^{2}=\mathrm{Ph}$ & terephthalaldehyde & 96 & 89 \\
\hline e & $\mathrm{R}^{1}=\mathrm{H} \quad \mathrm{R}^{2}=\mathrm{H}$ & Isophthalaldehyde & 94 & 87 \\
\hline$f$ & $\mathrm{R}^{1}=\mathrm{Me} \mathrm{R}^{2}=\mathrm{H}$ & Isophthalaldehyde & 94 & 90 \\
\hline g & $\mathrm{R}^{1}=\mathrm{H} \quad \mathrm{R}^{2}=\mathrm{Me}$ & Isophthalaldehyde & 94 & 89 \\
\hline h & $\mathrm{R}^{1}=\mathrm{H} \mathrm{R}^{2}=\mathrm{Ph}$ & Isophthalaldehyde & 93 & 88 \\
\hline $\mathbf{i}$ & $R^{1}=H \quad R^{2}=H$ & Phthalaldehyde & 94 & 89 \\
\hline j & $R^{1}=M e R^{2}=H$ & Phthalaldehyde & 93 & 87 \\
\hline k & $\mathrm{R}^{1}=\mathrm{H} \mathrm{R}^{2}=\mathrm{Me}$ & Phthalaldehyde & 91 & 86 \\
\hline 1 & $R^{1}=H R^{2}=P h$ & Phthalaldehyde & 94 & 89 \\
\hline
\end{tabular}

a 8 min at $850 \mathrm{~W}$. ${ }^{\text {b}}$ : Yield of isolated products. ${ }^{\mathrm{C}}$ : $3 \mathrm{~min}$ at $850 \mathrm{~W}$.

The structures of all the synthesized compounds were established on the basis of IR, ${ }^{1} \mathrm{HNMR},{ }^{13} \mathrm{C}-\mathrm{NMR}$ spectral data, and molecular weights were confirmed by high resolution mass spectrometry or elemental analysis. 
As an example, the ${ }^{1} \mathrm{H}-\mathrm{NMR}$ of compound $\mathbf{3 b}$ showed a singlet at $\delta 3.69$ integrating for 6 protons for the two methyl groups attached to nitrogen atoms; two singlets appeared at $\delta 5.95$, integrating for one proton, and $\delta 6.54$, integrating for two protons, are due to $\mathrm{H}_{10}$ and for hydrogens at position 2 of the indole structure, respectively. Two triplets appeared around $\delta 7.00(\mathrm{~J} 7 \mathrm{~Hz})$ and $7.24(\mathrm{~J} \mathrm{~Hz})$ each integrating for two protons attached at $\mathrm{H}_{5}$ and $\mathrm{H}_{6}$, respectively. A multiplet around $\delta$ 7.29-7.37 integrating for 4 protons is assigned to aromatic protons $\mathrm{H}_{4}$ and $\mathrm{H}_{7}$. Two doublets at $7.53(\mathrm{~J} 9 \mathrm{~Hz})$ and $7.80(\mathrm{~J} 9 \mathrm{~Hz})$ are due to $\mathrm{H}_{12}$ and $\mathrm{H}_{13}$ respectively. Finally, a singlet at $\delta 9.98$ integrating for one proton is assigned to $\mathrm{C}_{15}-\mathrm{H}$. Its ${ }^{13} \mathrm{C}-\mathrm{NMR}$ spectra showed 15 signals which corresponds to all magnetically inequivalent carbons. At $\delta 32.8$ and $\delta 40.4$ assigned to $C_{1}$ and $C_{10}$ respectively. The carbonyl carbon signal appeared at $\delta 192.2$ and the aromatic carbons appeared between $\delta$ 109.3 and $\delta 151.9$.

On the other hand, the ${ }^{1} \mathrm{H}-\mathrm{NMR}$ of compound $\mathbf{4 b}$ showed equivalent shifts, multiplicity and number of protons on each one signal for protons of methyl groups, $\mathrm{H}_{2}, \mathrm{H}_{10}, \mathrm{H}_{12}$ and $\mathrm{H}_{13}$ than compound $\mathbf{3 b}$ and the signal for proton the aldehyde is not observed. A singlet at $\delta 5.31$ is assigned to proton attached to nitrogen of the benzimidazole moiety and two multiplets around $\delta 6.70-7.40$ are assigned for the other aromatic protons. Its ${ }^{13} \mathrm{C}-\mathrm{NMR}$ spectra showed 18 signals which corresponds to all magnetically inequivalent carbons.

According to all of above, we think that our methodology to form compounds 3a-I has important green advantages over some previous mentioned synthetic methodologies for DIM derivatives, such as not use of solvent on reactions, compared with the use of dichloromethane $11,23,29,30,33,35$, chloroform ${ }^{13,32}$, acetonitrile ${ }^{12,38}$, ionic liquids ${ }^{27,28,29}$, not use of Broensted-Lowry and Lewis acid catalyst ${ }^{11,12,13,23,25,26,30,32,33,34,37,39}$, resins ${ }^{31,35,36,38}$, inert atmosphere ${ }^{26}$, and does not require long reaction times $\left(7-10 h^{29}, 24 h^{35}, 12-36 h^{13}\right)$, with the use of microwave energy for the activation of reactions. In addition, to obtain benzimidazole compounds $4 a-I$ we do not require any redox reagents, such as nitrobenzene ${ }^{16}$, sodium metabisulfite ${ }^{17}$, neither catalyst ${ }^{16,18,22}$ or solvent. ${ }^{18}$ In addition, all atomic economy values are excellent, 95.10-96.72\%.

\section{Conclusions}

We present straightforward synthetic methodology to generate novel hybrid diindolylmethylphenylbenzimidazole compounds. Because these reactions are conducted in the absence of solvents and catalysts and using microwave energy for the activation of the reactions, they provide the desired products under conditions that are within the context of green chemistry.

\section{Experimental Section}

General. Melting points were determined on a Buchi B-450 device and are uncorrected. The microwave monomode oven used was a Microwave Synthesis Reactor, Monowave 300, Anton Paar, employing sealed reaction vessels and the monitoring of the reaction mixture temperature was determined with an internal probe. The ${ }^{1} \mathrm{H}$ and ${ }^{13} \mathrm{C}$ NMR spectra were obtained from a Varian EM-390 (300 MHz) apparatus. Chemical shifts are given in ppm relative to TMS for $\mathrm{CDCl}_{3}$ or ppm relative to DMSO-d6 (Sigma-Aldrich) as mentioned in the corresponding spectral data (According to Gottlieb et al., the signals for DMSO and $\mathrm{H}_{2} \mathrm{O}$ present in DMSO- $d 6$ appear at 2.55 and $3.33 \mathrm{ppm}$, respectively). ${ }^{43}$ The following abbreviations are used: $s$, singlet; $d$, doublet; $t$, triplet; q, quartet; dd, doublet of doublet; $m$, multiplet. Mass spectrometry (MS) was performed on a JEOL JMSSX102A spectrometer by $\mathrm{EI}+$ at an ionization potential of $70 \mathrm{eV}$ and with $\mathrm{FAB}+$ techniques. IR spectra were 
obtained with a Perkin-Elmer 283B spectrophotometer, using either KBr tablet or film techniques. The following starting materials were commercially available: $1 \mathrm{H}$-indole, $\mathrm{N}$-methylindole, 2 -methylindole, 2-phenylindole, terephthalaldehyde, isophthalaldehyde, phthalaldehyde, o-phenylenediamine (all Sigma-Aldrich).

\section{Typical procedures}

(Formylphenyl)diindolylmethanes derivatives 3a-3I. In a microwave tube indole $(5.7141 \mathrm{mmol})$ and the dialdehyde $(2.8570 \mathrm{mmol}$ ) were added; this mixture was mixed well and irradiated with microwave energy (195 ${ }^{\circ} \mathrm{C}$ at $850 \mathrm{~W}$ ) for $8 \mathrm{~min}$. Then, once the mixture reaction cooled at room temperature, the corresponding reaction products were extracted with acetone and the solvent was removed at reduced pressure. All products were purified by recrystallization with a mixture of ethanol/water.

(Benzimidazol-2-yl)-3,3'-diindolylmethanes 4a-4l. In a microwave tube were added a (formylphenyl) diindolylmethane (3) $(1.4251 \mathrm{mmol})$ and $o$-phenylenediamine $(1.4251 \mathrm{mmol})$. The reagents were well blended and then irradiate with microwave energy for $3 \mathrm{~min}\left(195^{\circ} \mathrm{C}\right.$ at $\left.850 \mathrm{~W}\right)$. Microwave irradiation was performed at 1 minute intervals. The solids formed were dissolved in acetone and purified by preparative chromatography on silica gel using an eluting system of hexane/ethyl acetate (7:3). The products were scraped from the chromatographic plate and the solids were placed in a funnel. The silica gel was washed with hot ethanol, which was collected in a beaker in an ice bath; cold water was added for crystallization. In some cases, it was necessary to add a little cold acetone with water to achieve precipitation and the pure product was obtained as a sticky semisolid substance.

(4-Formylphenyl)(bis-(1H-indol-3-yl))methane (3a). (96\%), colorless crystals, mp $253-256{ }^{\circ} \mathrm{C}\left(\right.$ Lit $\left.^{23} \cdot 253-257^{\circ} \mathrm{C}\right)$; IR (KBr) 3404, 3051, 2835, 1691, 1602, 1574, 1415, 1337, 783, $\left.737 \mathrm{~cm}^{-1} ;{ }^{1} \mathrm{H} \mathrm{NMR} \mathrm{(300} \mathrm{MHz,} \mathrm{CDCl} 3, \mathrm{TMS}\right) \delta 5.96$ $(\mathrm{s}, 1 \mathrm{H}), 6.66(\mathrm{~s}, 2 \mathrm{H}), 6.97-7.04(\mathrm{t}, J 6 \mathrm{~Hz}, 2 \mathrm{H}), 7.25(\mathrm{t}, J 6 \mathrm{~Hz}, 2 \mathrm{H}), 7.36(\mathrm{t}, J 6 \mathrm{~Hz}, 2 \mathrm{H}), 7.50-7.52(\mathrm{~d}, J 7 \mathrm{~Hz}, 2 \mathrm{H})$, 7.78-7.81 (d, J $8 \mathrm{~Hz}, 2 \mathrm{H}), 7.97\left(\mathrm{~s}, 2 \mathrm{H}, \mathrm{NH}, \mathrm{D}_{2} \mathrm{O}\right), 8.01(\mathrm{~s}, 2 \mathrm{H}) 9.97(\mathrm{~s}, 1 \mathrm{H}) \mathrm{ppm} ;{ }^{13} \mathrm{C} \mathrm{NMR}\left(75 \mathrm{MHz}, \mathrm{CDCl}_{3}, \mathrm{TMS}\right) \delta$ $40.4,109.3,117.1,118.9,119.8,121.7,123.6,127.2,129.4,129.9,134.8,137.5,151.9,192.2 \mathrm{ppm} ; \mathrm{MS}(\mathrm{EI}) \mathrm{m} / \mathrm{z}$ (\%) $350(\mathrm{M}+, 100) . \mathrm{ESI}+\mathrm{MS}[1]$ Calc. 351.14943, Found 351.14995.

(4-Formylphenyl)(bis(1-methyl-1H-indol-3-yl))methane (3b). (93\%), colorless crystals, $m p$ 169-171 ${ }^{\circ} \mathrm{C}$ (Lit. ${ }^{39}$ $\left.170-172{ }^{\circ} \mathrm{C}\right)$; IR (KBr) 3047, 2910, 2878, 1698, 1468, 1610, 1546, 788, $737 \mathrm{~cm}^{-1},{ }^{1} \mathrm{H}$ NMR $\left(300 \mathrm{MHz}, \mathrm{CDCl}_{3}, \mathrm{TMS}\right) \delta$ $3.69(\mathrm{~s}, 6 \mathrm{H}), 5.95(\mathrm{~s}, 1 \mathrm{H}), 6.54(\mathrm{~s}, 2 \mathrm{H}), 7.00\left(\mathrm{t}, J 7 \mathrm{~Hz}, 2 \mathrm{H}^{\prime}\right), 7.24(\mathrm{t}, J 7 \mathrm{~Hz}, 2 \mathrm{H}), 7.29-7.37(\mathrm{~m}, 4 \mathrm{H}), 7.53(\mathrm{~d}, J 9 \mathrm{~Hz}$, $2 \mathrm{H}), 7.80$ (d, J $9 \mathrm{~Hz}, 2 \mathrm{H}), 9.98(\mathrm{~s}, \mathrm{H}) \mathrm{ppm} ;{ }^{13} \mathrm{C} \mathrm{NMR}\left(75 \mathrm{MHz}, \mathrm{CDCl}_{3}, \mathrm{TMS}\right) \delta 32.8,40.4,109.3,117.1,118.9,119.8$, $121.7,127.2,128.3,129.4,129.9,134.8,137.5,151.9,192.2$ ppm. MS (EI) m/z (\%) 378 (M+, 100). ESI+ MS[1] Calc. 379.18104 , Found 379.18068.

(4-Formylphenyl)(bis(2-methyl-1H-indol-3-yl))methane (3c). (94\%), colorless crystals, $m p$ 127-128 ${ }^{\circ} \mathrm{C} ; \mathrm{IR}(\mathrm{KBr})$ $3400,3302,3052,2920,2853,1684,1458,1601,1573,1302,829,811,740 \mathrm{~cm}^{-1}$; ${ }^{1} \mathrm{H} \mathrm{NMR}\left(300 \mathrm{MHz} \mathrm{CDCl}_{3}\right.$, TMS) $\delta 2.08(\mathrm{~s}, 6 \mathrm{H}), 6.05(\mathrm{~s}, 1 \mathrm{H}), 6.83-6.95(\mathrm{~m}, 4 \mathrm{H}), 7.05(\mathrm{t}, J 8.1 \mathrm{~Hz}, 2 \mathrm{H}), 7.26(\mathrm{~d}, J 7.5 \mathrm{~Hz}, 2 \mathrm{H}), 7.43(\mathrm{~d}, J 8.1 \mathrm{~Hz}$, $2 \mathrm{H}), 7.76(\mathrm{~d}, J 8.1 \mathrm{~Hz}, 2 \mathrm{H}), 7.80\left(\mathrm{~s}, 2 \mathrm{H}, \mathrm{NH}, \mathrm{D}_{2} \mathrm{O}\right), 9.98(\mathrm{~s}, 1 \mathrm{H}) \mathrm{ppm} ;{ }^{13} \mathrm{C} \mathrm{NMR}\left(75 \mathrm{MHz}, \mathrm{CDCl}_{3}, \mathrm{TMS}\right) \delta 12.4,39.9$, $110.2,112.0,119.0,120.6,128.7,129.7,129.8,132.3,134.5,135.2,151.9,192.2$ ppm; MS(EI) $\mathrm{m} / z$ (\%) $378\left(\mathrm{M}^{+}\right.$, 100). ESI+ MS[1] Calc. 379.18104, Found 379.18073.

(4-Formylphenyl)(bis(2-phenyl-1H-indol-3-yl))methane (3d). (96\%), colorless crystals, $m p$ 260-262 ${ }^{\circ} \mathrm{C} ; \mathrm{IR}(\mathrm{KBr})$ 3399, 3056, 3027, 1694, 1449,1602, 1575, 1553, 1336, 773, 733, $696 \mathrm{~cm}^{-1} ;{ }^{1} \mathrm{H}$ NMR (300 MHz; CDCl, $\left.\mathrm{TMS}\right) \delta$ $5.97(\mathrm{~s}, 1 \mathrm{H}), 6.94-7.03(\mathrm{~m}, 4 \mathrm{H}), 7.15-7.37(\mathrm{~m}, 14 \mathrm{H}), 7.44(\mathrm{t}, J 9 \mathrm{~Hz}, 2 \mathrm{H}), 7.62-7.65(\mathrm{~d}, J 9 \mathrm{~Hz}, 2 \mathrm{H}), 7.73-7.76(\mathrm{~d}, J 9$ $\mathrm{Hz}, 2 \mathrm{H}), 7.86$ (s, 2H), 9.95 (s, 1H) ppm; ${ }^{13} \mathrm{C} \mathrm{NMR}\left(75 \mathrm{MHz} ; \mathrm{CDCl}_{3}, \mathrm{TMS}\right) \delta 39.9,109.0,109.2,117.4,118.8,119.8$, $121.6,127.2,127.5,128.3,128.9,130.2,134.9,136.5,137.5,145.8,192.7$ ppm, MS(EI) m/z (\%) 502 (M+, 8). $\mathrm{FAB}+$ Calc. 502.2045, Found 502.2049. 
(3-Formylphenyl)(bis(1H-indol-3-yl))methane (3e). (94\%), colorless crystals, $m p$ 176-178 ${ }^{\circ} \mathrm{C}$; IR (KBr) 3399, 3051, 2908, 1686, 1451, 1602, 1310, 843, $737 \mathrm{~cm}^{-1}$; ${ }^{1} \mathrm{H}$ NMR (300 MHz, CDCl, $\left.\mathrm{TMS}\right) 6.07$ (s, $1 \mathrm{H}$ ), 6.89 (t, J $5 \mathrm{~Hz}, 2 \mathrm{H}$ ), 7.06 (t, J $6 \mathrm{~Hz}, 2 \mathrm{H}), 7.29-7.37(\mathrm{~m}, 4 \mathrm{H}), 7.59$ (d, J $3 \mathrm{~Hz}, 2 \mathrm{H}), 7.84$ (d, J $9 \mathrm{~Hz}, 2 \mathrm{H}), 8.07-8.09(\mathrm{~d}, J 6 \mathrm{~Hz}, 2 \mathrm{H}), 8.17(\mathrm{~s}$, $2 \mathrm{H}, \mathrm{NH}, \mathrm{D}_{2} \mathrm{O}$ ), 9.98 (s, 1H) ppm; ${ }^{13} \mathrm{C}$ NMR (75 MHz, CDCl $\left.3, \mathrm{TMS}\right) \delta 34.7,108.5,111.0,117.4,119.3,119.5,122.6$, 123, 124.2, 126.5, 127.6, 130.9, 132.2, 136.5, 137.4, 192.2 ppm; MS(EI) m/z (\%) 350 (M+, 100). ESI+ MS[1] Calc. 351.4974, Found 351.14890.

(3-Formylphenyl)(bis(1-methyl-1H-indol-3-yl))methane (3f). (94\%), colorless crystals, $m p$ 139-142 ${ }^{\circ} \mathrm{C} ; \mathrm{IR}(\mathrm{KBr})$ 3050; 2931; 2910; 2838; 2821; 1687; 1469; 1687; 1610; 1582; 1546); 1327; 794; $737 \mathrm{~cm}^{-1} ;{ }^{1} \mathrm{H} \mathrm{NMR} \mathrm{(300} \mathrm{MHz,}$ $\left.\mathrm{CDCl}_{3}, \mathrm{TMS}\right) \delta 3.70\left(\mathrm{~s}, 6 \mathrm{H}, 2 \mathrm{CH}_{3}\right), 5.97(\mathrm{~s}, 1 \mathrm{H}), 6.53\left(\mathrm{~s}, 2 \mathrm{H}^{\prime}\right), 7.00(\mathrm{t}, J 6 \mathrm{~Hz}, 2 \mathrm{H}), 7.19-7.30(\mathrm{~m}, 6 \mathrm{H}), 7.37(\mathrm{t}, J 6 \mathrm{~Hz}$, $1 \mathrm{H}), 7.64(\mathrm{~d}, J 6 \mathrm{~Hz}, 1 \mathrm{H}), 7.74(\mathrm{~d}, J 6 \mathrm{~Hz}, 1 \mathrm{H}), 7.86(\mathrm{~s}, 1 \mathrm{H}), 9.95(\mathrm{~s}, 1 \mathrm{H}) \mathrm{ppm} ;{ }^{13} \mathrm{C} \mathrm{NMR}(75 \mathrm{MHz}, \mathrm{CDCl}, \mathrm{TMS}) \delta 32.4$, 40.3, 109.2, 109.4, 117.1, 118.9, 119.7, 121.3, 127.7, 128.4, 129.8, 134.7, 137.4, 144.5, 146.0, 192.0 ppm, MS(EI) $\mathrm{m} / \mathrm{z}(\%) 378\left(\mathrm{M}^{+}, 73\right) . \mathrm{FAB}+$ Calc. 378.1732, Found 378.1729.

(3-Formylphenyl)(bis(2-methyl-1H-indol-3-yl))methane (3g). (94\%), colorless crystals, $m p$ 140-142 ${ }^{\circ} \mathrm{C} ; \mathrm{IR}(\mathrm{KBr})$, $3404,3050,2838,1686,1454,1601,1515,1336,792,739 \mathrm{~cm}^{-1} ;{ }^{1} \mathrm{H} \mathrm{NMR}\left(300 \mathrm{MHz}, \mathrm{CDCl}_{3}, \mathrm{TMS}\right) \delta 2.12(\mathrm{~s}, 6 \mathrm{H}$, $2 \mathrm{CH} 3), 6.00(\mathrm{~s}, 1 \mathrm{H}), 6.80(\mathrm{t}, J 9 \mathrm{~Hz}, 3 \mathrm{H}), 6.90(\mathrm{~d}, J 6 \mathrm{~Hz}, 2 \mathrm{H}), 6.98(\mathrm{t}, J 9 \mathrm{~Hz}, 3 \mathrm{H}), 7.23(\mathrm{~d}, J 9 \mathrm{~Hz}, 2 \mathrm{H}), 7.40(\mathrm{~d}, J 9 \mathrm{~Hz}$, $2 \mathrm{H}), 7.70(\mathrm{~d}, J 6 \mathrm{~Hz}, 2 \mathrm{H}), 9.93(\mathrm{~s}, 1 \mathrm{H}) \mathrm{ppm} ;{ }^{13} \mathrm{C} \mathrm{NMR}\left(75 \mathrm{MHz}, \mathrm{CDCl}_{3}, \mathrm{TMS}\right) \delta 12.4,40.2,110.2,112.1,119.3$, $119.9,120.6,127.2,128.2,128.5,129.2,132.3,134.5,135.2,136.8,137.6,192.2$ ppm; MS(EI) $\mathrm{m} / z$ (\%) $378\left(\mathrm{M}^{+}\right.$, 100). ESI+ MS[1] Calc. 379.18104, Found 379.18038.

(3-Formylphenyl)(bis(2-phenyl-1H-indol-3-yl))methane (3h). (93\%), colorless crystals, mp 202-205 ${ }^{\circ} \mathrm{C} ; \mathrm{IR}(\mathrm{KBr})$ $3424,3395,3053,1686,1598,1485,1339,1310,843,740,698 \mathrm{~cm}^{-1} ;{ }^{1} \mathrm{H}$ NMR $\left(300 \mathrm{MHz}, \mathrm{CDCl}_{3}, \mathrm{TMS}\right) \delta 5.99(\mathrm{~s}$, $1 \mathrm{H}), 6.65-6.92(\mathrm{~m}, J 6 \mathrm{~Hz}, 4 \mathrm{H}), 7.01(\mathrm{t}, J 6 \mathrm{~Hz}, 2 \mathrm{H}), 7.15-7.39(\mathrm{~m}, 18 \mathrm{H}), 9.98(\mathrm{~s}, 1 \mathrm{H}) \mathrm{ppm} ;{ }^{13} \mathrm{C} \mathrm{NMR}\left(75 \mathrm{MHz}, \mathrm{CDCl}_{3}\right.$, TMS) $\delta 40.2,109.0,109.2,119.8,120.1,121.2,121.6,127.2,127.5,128.2,128.3,128.9,129.4,129.6,130.2$, 134.9, 136.5, 137. 4, 137.4, 192.7 ppm; MS(EI) m/z (\%) 502 ( $\left.\mathrm{M}^{+}, 7\right)$. ESI+ MS[1] Calc. 503.21234, Found 503.21105.

(2-Formylphenyl)(bis(1H-indol-3-yl))methane (3i). (94\%), colorless crystals, $m p$ 117-118 ${ }^{\circ} \mathrm{C}$; IR ( $\left.\mathrm{KBr}\right) 3400,3051$, 2957, 2919, 1702, 1452, 1608, 1541, 1317, 875, 834, $739 \mathrm{~cm}^{-1} ;{ }^{1} \mathrm{H}$ NMR (300 MHz, CDCl 3 TMS) $\delta 5.83$ (s $\left.1 \mathrm{H}\right)$, $6.56(\mathrm{~s}, 2 \mathrm{H}), 6.96\left(\mathrm{t}, J 15 \mathrm{~Hz}, 2 \mathrm{H}^{\prime}\right), 7.08-7.37(\mathrm{~m}, 10 \mathrm{H}), 7.76(\mathrm{~s}, 2 \mathrm{H}), 9.99(\mathrm{~s}, 1 \mathrm{H}) \mathrm{ppm} ;{ }^{13} \mathrm{C} \mathrm{NMR}\left(75 \mathrm{MHz}, \mathrm{CDCl}_{3}\right.$, TMS) $\delta$ 39.9, 109.9, 110.8, 116.6, 119.5, 119.7, 131.7, 123.4, 125.9, 126.8, 128.0, 128.5, 130.4, 137.7, 143.8, 193.4 ppm; MS(EI) m/z (\%) $350\left(\mathrm{M}^{+}, 8\right)$. ESI+ MS[1] Calc. 350.14191, Found 350.14211.

(2-Formylphenyl)(bis(1-methyl-1H-indol-3-yl))methane (3j). (93\%), colorless crystals, $m p$ 179-181 ${ }^{\circ} \mathrm{C}$; IR (KBr) $3050,3019,2928,2879,2841,1473,1539,1344,1317,866,828,740 \mathrm{~cm}^{-1} ;{ }^{1} \mathrm{H}$ NMR $\left(300 \mathrm{MHz}, \mathrm{CDCl}_{3}, \mathrm{TMS}\right) \delta$ $3.69(\mathrm{~s}, 6 \mathrm{H}, 2 \mathrm{CH} 3), 5.95(\mathrm{~s}, 1 \mathrm{H}), 6.53(\mathrm{~s}, 2 \mathrm{H}), 7.00(\mathrm{t}, J 6 \mathrm{~Hz}, 2 \mathrm{H}), 7.25-7.33(\mathrm{~m}, 6 \mathrm{H}), 7.52(\mathrm{~d}, J 9 \mathrm{~Hz}, 2 \mathrm{H}), 7.78(\mathrm{~d}, J 9$

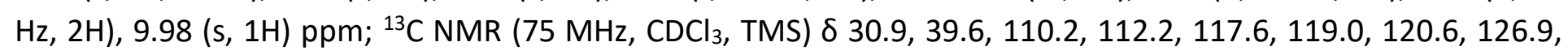
127.6, 128.3, 129.1, 130.2, 134.5, 135.2, 137.1, 138.9, 191.8 ppm; MS(EI) m/z (\%) 378 (M+, 5). ESI+ MS[1] Calc. 379.18104 , Found 379.17955.

(2-Formylphenyl)(bis(2-methyl-1H-indol-3-yl))methane (3k). (91\%), colorless crystals, $m p$ 218-220 ${ }^{\circ} \mathrm{C} ; \mathrm{IR}(\mathrm{KBr})$ $3279,3050,2931,2870,2827,1687,1468,1609,1583,1327,794,767,737 \mathrm{~cm}^{-1}$; ${ }^{1} \mathrm{H} \mathrm{NMR}\left(300 \mathrm{MHz}^{2} \mathrm{CDCl}_{3}\right.$, TMS) $\delta 1.96(\mathrm{~s}, 6 \mathrm{H}, 2 \mathrm{CH}), 6.00(\mathrm{~s}, 1 \mathrm{H}), 6.87(\mathrm{~s}, 1 \mathrm{H}), 6.93(\mathrm{t}, J 7.5 \mathrm{~Hz}, 1 \mathrm{H}), 7.13(\mathrm{t}, J 7.8 \mathrm{~Hz}, 2 \mathrm{H}), 7.33(\mathrm{~d}, J 7.8 \mathrm{~Hz}$, $1 \mathrm{H}), 7.40(\mathrm{~d}, J 8.1 \mathrm{~Hz}, 1 \mathrm{H}), 7.53(\mathrm{~d}, J 8 \mathrm{~Hz}, 2 \mathrm{H}), 7.71-7.89(\mathrm{~m}, 6 \mathrm{H}), 9.94(\mathrm{~s}, 1 \mathrm{H}) \mathrm{ppm} ;{ }^{13} \mathrm{C} \mathrm{NMR}\left(75 \mathrm{MHz}, \mathrm{CDCl}_{3}\right.$, TMS) $\delta 12.4,31.3,110.6,112.8,118.7,121.3,122.8,126.5,127.9,129.3,132.2,134.9,136.4,138.2,139.1$, 141.9, 191.5 ppm; MS(EI) m/z (\%) 378 (M+, 100). ESI+ MS[1] Calc. 378.17321, Found 378.17222.

(2-Formylphenyl)(bis(2-phenyl-1H-indol-3-yl))methane (3I). (94\%), colorless crystals, $m p$ 100-103 ${ }^{\circ} \mathrm{C} ; \mathrm{IR}(\mathrm{KBr})$ $3413,3387,3335,3057,3028,1684,1595,1575,1425,1342,765,744 \mathrm{~cm}^{-1} ;{ }^{1} \mathrm{H}$ NMR $\left(300 \mathrm{MHz}, \mathrm{CDCl}_{3}, \mathrm{TMS}\right) \delta$ $5.52(\mathrm{~s}, 1 \mathrm{H}), 6.85(\mathrm{t}, J 9 \mathrm{~Hz}, 2 \mathrm{H}), 7.07(\mathrm{t}, J 9 \mathrm{~Hz}, 2 \mathrm{H}), 7.19-7.42(\mathrm{~m}, 16 \mathrm{H}), 7.54(\mathrm{~d}, J 9 \mathrm{~Hz}, 2 \mathrm{H}), 7.98(\mathrm{~s}, 2 \mathrm{H}), 9.90(\mathrm{~s}$, 
1H) ppm; ${ }^{13} \mathrm{C} \mathrm{NMR} \mathrm{(75} \mathrm{MHz,} \mathrm{CDCl}_{3}$, TMS) $\delta 42.9,108.9,111.1,118.9,119.9,121.6,123.3,126.3,127.5,127.5$, $128.2,129.3,129.8,131.2,133.3,134.2,135.2,137.5,138.8,191.7$ ppm; MS(FAB+) m/z (\%) 503 (M+1, 4). ESI+ MS[1] Calc. 503.21234, Found 503.21120.

2-(4-(Bis(1H-indol-3-yl)methyl)phenyl)-1H-benzimidazole. (88\%), colorless crystals, $m p$ 206-208 ${ }^{\circ} \mathrm{C} ; \mathrm{IR}(\mathrm{KBr})$ $3410,3051,2955,2918,2850,1704,1454,1247,1610,1337,868,805,738 \mathrm{~cm}^{-1} ;{ }^{1} \mathrm{H}$ NMR $\left(300 \mathrm{MHz}\right.$, DMSO-d $d_{6}$ ref. DMSO- $\left.d_{6}\right) \delta 5.81(\mathrm{~s} 1 \mathrm{H}), 6.39-7.27(\mathrm{~m}, \mathrm{H} 12)$ 7.86-8.04 (d, J $\left.6 \mathrm{~Hz}, 2 \mathrm{H}\right), 8.01-8.29(\mathrm{~m} 2 \mathrm{H}), 8.59(\mathrm{~s}, 2 \mathrm{H}), 10.67(\mathrm{~s}$, $1 \mathrm{H}, \mathrm{NH}), 10.72(\mathrm{~d}, 1 \mathrm{H}, \mathrm{NH}), 10.82(\mathrm{~s}, 1 \mathrm{H}, \mathrm{NH}), \mathrm{ppm} ;{ }^{13} \mathrm{C} \mathrm{NMR}\left(75 \mathrm{MHz}, \mathrm{DMSO}-d_{6}\right.$, ref. DMSO-d $) \delta 41.0,111.1$, $112.1,115.2,118.6,119.0,121.6,123.2,125.4,127.3,129.4,131.7,136.3,138.2,141.5,152.2 \mathrm{ppm} ; \mathrm{MS}(\mathrm{EI}) \mathrm{m} / \mathrm{z}$ (\%) $438\left(\mathrm{M}^{+}, 13\right) . \mathrm{ESI}+\mathrm{MS}[1]$ Calc. 439.19227, Found 439.19143.

2-(4-(Bis(1-methyl-1H-indol-3-yl)methyl)phenyl)-1H-benzimidazole (4b). (92\%), colorless crystals, $\mathrm{mp} 108-111$ ${ }^{\circ} \mathrm{C} . \mathrm{IR}(\mathrm{KBr}) 3054,3011,3011,2960,2907,2877,2851,1698,1437,1280,1615,1567,1319,846,738 \mathrm{~cm}^{-1} ;{ }^{1} \mathrm{H}$ NMR (300 MHz, DMSO- $d_{6}$, ref. DMSO- $\left.d_{6}\right) \delta 3.73(\mathrm{~s}, 6 \mathrm{H}), 5.31(\mathrm{~s}, 1 \mathrm{H}, \mathrm{NH}), 5.94(\mathrm{~s}, 1 \mathrm{H}), 6.38(\mathrm{t}, J 3 \circ 6 \mathrm{~Hz}, 2 \mathrm{H})$, $6.73(\mathrm{q}, J 6 \mathrm{~Hz}, 2 \mathrm{H}), 6.88-6.97(\mathrm{~m}, 4 \mathrm{H}), 7.33-7.50(\mathrm{~m}, 6 \mathrm{H}), 7.89-7.92(\mathrm{~d}, J 9 \mathrm{~Hz}, 2 \mathrm{H}), 8.13(\mathrm{~s}, 2 \mathrm{H}) \mathrm{ppm} ;{ }^{13} \mathrm{C} \mathrm{NMR}(75$ $\mathrm{MHz}, \mathrm{DMSO}-d_{6}$, ref. DMSO- $\left.d_{6}\right) \delta 32.7,56.1,108.0,110.1,114.9,116.5,117.7,118.8,119.6,121.6,127.2,128.4$, 129.0; 134.9, 135.4, 136.1, 148.4, 155.9 ppm; MS(EI) $m / z(\%) 466$ ( $\left.\mathrm{M}^{+}, 7\right)$. FAB+ Calc. 467.2236, Found 467.2228.

2-(4-(Bis(2-methyl-1H-indol-3-yl)methyl)phenyl)-1H-benzimidazole (4c). (94\%), colorless crystals, $\mathrm{mp} 235-237$ ${ }^{\circ} \mathrm{C}$; IR (KBr) 3379, 3051, 2955, 2918, 2850, 1652, 1462, 1248, 1560, 1409, 1342, 918, 869, $738 \mathrm{~cm}^{-1} ;{ }^{1} \mathrm{H} \mathrm{NMR}(300$ $\mathrm{MHz}, \mathrm{DMSO}-d_{6}$, ref. DMSO- $\left.d_{6}\right) \delta 2.13(\mathrm{~s}, 6 \mathrm{H}), 4.41(\mathrm{~s}, 1 \mathrm{H}), 6.40(\mathrm{~d}, J 6 \mathrm{~Hz}, 2 \mathrm{H}), 6.53(\mathrm{~d}, J 6 \mathrm{~Hz}, 2 \mathrm{H}), 6.71-6.74(\mathrm{~d}, J 9$ $\mathrm{Hz}, 2 \mathrm{H}), 6.90(\mathrm{t}, J 9 \mathrm{~Hz}, 2 \mathrm{H}), 7.21-7.23(\mathrm{~d}, J 6 \mathrm{~Hz}, 3 \mathrm{H}), 7.58-7.91(\mathrm{~m}, 4 \mathrm{H}), 8.09(\mathrm{~d}, J 6 \mathrm{~Hz}, 2 \mathrm{H}), 10.75(\mathrm{~s}, 1 \mathrm{H}, \mathrm{NH})$, 10.84 (s $1 \mathrm{H}, \mathrm{NH}) \mathrm{ppm} ;{ }^{13} \mathrm{C}$ NMR $\left(75 \mathrm{MHz}\right.$, DMSO-d $d_{6}$, ref. DMSO-d 6 ) $\delta 12.8,41.6,110.8,112.1,115.2,115.3$, $118.4,119.6,121.3,123.5,125.2,127.4,129.4,131.0,131.3,141.1,153.9$ ppm; MS(EI) m/z (\%) $466\left(M^{+}, 18\right)$. ESI+ MS[1] Calc. 467.22357, Found 467.22209.

2-(4-(Bis(2-phenyl-1H-indol-3-yl)methyl)phenyl)-1H-benzimidazole (4d). (89\%), colorless crystals, mp $150-152$ ${ }^{\circ} \mathrm{C}$; IR (KBr) 3464, 3390, 3049, 2980, 2832, 1600, 1487, 1450, 822, 739, $698 \mathrm{~cm}^{-1}$; ${ }^{1} \mathrm{H}$ NMR (300 MHz, DMSO-d6, ref. DMSO- $\left.d_{6}\right) \delta 5.58(\mathrm{~s}, 1 \mathrm{H}), 6.97-7.10(\mathrm{~m}, 4 \mathrm{H}), 7.21-7.41(\mathrm{~m}, 10 \mathrm{H}), 7.53-7.71(\mathrm{~m}, 4 \mathrm{H}), 7.87(\mathrm{~m}, 2 \mathrm{H}), 8.11-8.23$ $(\mathrm{m}, 2 \mathrm{H}), 8.32(\mathrm{~d}, J 9 \mathrm{~Hz}, 2 \mathrm{H}), 8.66(\mathrm{~d}, J 6 \mathrm{~Hz}, 2 \mathrm{H}), 11.38(\mathrm{~s}, 2 \mathrm{H}, \mathrm{NH}), 11.46(\mathrm{~s}, 1 \mathrm{H}, \mathrm{NH}) \mathrm{ppm} ;{ }^{13} \mathrm{C} \mathrm{NMR}(75 \mathrm{MHz}$; DMSO- $d_{6}$, ref. DMSO- $\left.d_{6}\right) \delta 41.4,108.1,111.4,115.8,118.4,118.6,119.7,121.7,123.3,125.0,127.9,128.8$, $129.5,129.8,131.3,133.1,136.8,138.5,141.6,153.1$ ppm; MS(EI) $m / z$ (\%) $590\left(\mathrm{M}^{+}, 2\right)$. Elemental analysis: Calc. C, 85.40; H, 5.12; N, 9.48, Found. C, 85.38; H, 5.10; N, 9.43.

2-(3-(Bis(1H-indol-3-yl)methyl)phenyl)-1H-benzimidazole (4e). (87\%), colorless crystals, $m p$ 195-197 ${ }^{\circ} \mathrm{C}$; IR (KBr) $3512,3383,3361,2955,2918,2850,1620,1590,1466,1248,1210,869,804,722 \mathrm{~cm}^{-1} ;{ }^{1} \mathrm{H} \mathrm{NMR}(300 \mathrm{MHz}$, DMSO- $d_{6}$, ref. DMSO- $\left.d_{6}\right) \delta 4.42(\mathrm{~s} \mathrm{brd}, 2 \mathrm{H}, \mathrm{NH}), 5.75(\mathrm{~s}, 1 \mathrm{H}), 6.36-6.39(\mathrm{~m}, 2 \mathrm{H}), 6.49-6.52(\mathrm{~m}, 4 \mathrm{H}), 6.88(\mathrm{~d}, J 6 \mathrm{~Hz}$, $2 \mathrm{H}), 7.13(\mathrm{~d}, J 6 \mathrm{~Hz}, 2 \mathrm{H}), 7.20(\mathrm{~d}, J 6 \mathrm{~Hz}, 2 \mathrm{H}), 7.39-7.49(\mathrm{~m}, 4 \mathrm{H}), 7.80(\mathrm{~d}, J 6 \mathrm{~Hz}, 1 \mathrm{H}), 8.09(\mathrm{~s}, 1 \mathrm{H}), 10.65(\mathrm{~s}, 1 \mathrm{H})$ ppm; ${ }^{13} \mathrm{C}$ NMR (75 MHz, DMSO- $d_{6}$, ref. DMSO-d $)_{6} \delta 60.1,110.4,110.8,112.7,118.1,119.1,119.9,121.6,123.1$, $126.3,126.4,128.6,130.0,132.2,135.1,136.7,137.9,144.2,152.1$ ppm; MS(EI) $m / z(\%) 438$ (M+, 2). ESI+ MS[1] Calc. 439.19227, Found 439.19143.

2-(3-(Bis(1-methyl-1H-indol-3-yl)methyl)phenyl)-1H-benzimidazole (4f). (90\%), colorless crystals, $m p$ 139-142 ${ }^{\circ} \mathrm{C}$; IR (KBr) 3330, 3054, 3006, 2962, 2631, 2880, 2823, 1708, 1638, 1599, 1474, 1370, $883 \mathrm{~cm}^{-1} ;{ }^{1} \mathrm{H}$ NMR (300 $\mathrm{MHz}, \mathrm{DMSO}-d_{6}$, ref. DMSO-d $d_{6} \delta 3.63(\mathrm{~s}, 3 \mathrm{H}), 3.72(\mathrm{~s}, 3 \mathrm{H}), 5.14(\mathrm{~s}, 1 \mathrm{H}, \mathrm{NH}), 5.76(\mathrm{~s}, 1 \mathrm{H}), 6.00(\mathrm{~d}, J 3 \mathrm{~Hz}, 2 \mathrm{H}), 6.69$ $(\mathrm{s}, 2 \mathrm{H}), 6.87-6.97(\mathrm{~m}, 3 \mathrm{H}), 7.11-7.27(\mathrm{~m}, 3 \mathrm{H}), 7.38(\mathrm{t}, J 9 \mathrm{~Hz}, 2 \mathrm{H}), 7.50(\mathrm{~s}, 2 \mathrm{H}), 7.71-7.85(\mathrm{~m}, 2 \mathrm{H}), 7.90(\mathrm{~s}, 1 \mathrm{H}), 8.05$ (s, $1 \mathrm{H}) \mathrm{ppm} ;{ }^{13} \mathrm{C}$ NMR $\left(75 \mathrm{MHz} \text {, DMSO- } d_{6} \text {, ref. DMSO-d }\right)_{6} \delta 32.7,56.1,108.5,110.0,111.7,114.9,116.6,117.0$, $117.6,118.4,118.8,119.5,119.6,121.3,121.7,122.1,126.3,127.3,128.1,128.5,137.2,144.6 \mathrm{ppm} ; \mathrm{MS}(\mathrm{EI}) \mathrm{m} / \mathrm{z}$ (\%) $466\left(\mathrm{M}^{+}, 58\right) . \mathrm{ESI}+\mathrm{MS}[1]$ Calc. 467.22357, Found 467.22322. 
2-(3-(Bis(2-methyl-1H-indol-3-yl)methyl)phenyl)-1H-benzimidazole (4g). (89\%), colorless crystals, $m p$ 114-116 ${ }^{\circ} \mathrm{C}$; IR (KBr) 3390; 3053, 2914, 2746, 1696, 1607, 1575, 1454, 1437, 1338, 845, 802, $730 \mathrm{~cm}^{-1} ;{ }^{1} \mathrm{H} \mathrm{NMR}$ (300 MHz, DMSO- $d_{6}$, ref. DMSO- $\left.d_{6}\right) \delta 2.10(\mathrm{~s}, 6 \mathrm{H}), 5.17(\mathrm{~s}, 1 \mathrm{H}, \mathrm{NH}), 5.95(\mathrm{~d}, J 9 \mathrm{~Hz}, 2 \mathrm{H}), 6.43(\mathrm{~s}, 2 \mathrm{H}), 6.60-6.68(\mathrm{q}, J 9 \mathrm{~Hz}, 2 \mathrm{H})$, $6.87(\mathrm{t}, J 9 \mathrm{~Hz}, 2 \mathrm{H}), 7.00-7.09(\mathrm{~m}, 2 \mathrm{H}), 7.17-7.26(\mathrm{~m}, 3 \mathrm{H}), 7.33-7.40(\mathrm{~m}, 2 \mathrm{H}), 8.72(\mathrm{~d}, J 12 \mathrm{~Hz}, 2 \mathrm{H}), 10.87(\mathrm{~s}, 1 \mathrm{H}$, $\mathrm{NH}), 10.93(\mathrm{~d}, 1 \mathrm{H}, \mathrm{NH}) \mathrm{ppm} ;{ }^{13} \mathrm{C} \mathrm{NMR}\left(75 \mathrm{MHz}, \mathrm{DMSO}-d_{6}\right.$, ref. DMSO- $\left.d_{6}\right) \delta 22.0,41.6,111.1,112.1,115.2,118.6$, $119.7,121.3,123.1,127.4,128.6,129.2,129.7,130.2,136.2,137.4,138.4,141.3,153.0 \mathrm{ppm} ; \mathrm{MS}(\mathrm{FAB}+) \mathrm{m} / \mathrm{z}(\%)$ $467\left(\mathrm{M}^{+}+1,4\right) . \mathrm{FAB}+$ Calc. 467.2236, Found 467.2228.

2-(3-(Bis(2-phenyl-1H-indol-3-yl)methyl)phenyl)-1H-benzimidazole (4h). (88\%), colorless crystals, $m p$ 221-224 ${ }^{\circ} \mathrm{C}$; IR (KBr) 3409, 3052, 2975, 1602, 1485, 1449, 800, 739, $695 \mathrm{~cm}^{-1} ;{ }^{1} \mathrm{H}$ NMR (300 MHz, DMSO- $d_{6}$, ref. DMSO- $\left.d_{6}\right)$ $\delta 5.73(\mathrm{~s}, 1 \mathrm{H}), 6.71(\mathrm{t}, J 6 \mathrm{~Hz}, 2 \mathrm{H}), 6.96(\mathrm{t}, J 6 \mathrm{~Hz}, 6 \mathrm{H}), 7.09(\mathrm{~m}, 6 \mathrm{H}), 7.23(\mathrm{~d}, J 6 \mathrm{~Hz}, 9 \mathrm{H}), 7.3(\mathrm{~s}, 1 \mathrm{H}), 7.38-7.41(\mathrm{~d}, J$ $9 \mathrm{~Hz}, 2 \mathrm{H}), 11.31(\mathrm{~s}, 1 \mathrm{H}, \mathrm{NH}), 11.41(\mathrm{~d}, 1 \mathrm{H}, \mathrm{NH}), 11.47(\mathrm{~s}, 1 \mathrm{H}, \mathrm{NH}), \mathrm{ppm} ;{ }^{13} \mathrm{C}$ NMR $\left(75 \mathrm{MHz}, \mathrm{DMSO}-d_{6}\right.$, ref. DMSO$\left.d_{6}\right) \delta 56.4,108.0,111.6,115.0,117.3,119.3,121.0,121.3,127.2,127.3,128.4,128.6,130.1,133.0,134.3,135.3$, 136.6, 137.2, 146.7 ppm; MS(EI) $m / z(\%) 590\left(\mathrm{M}^{+}, 11\right)$. Elemental analysis: Calc. C, 85.40; H, 5.12; N, 9.48, Found. C, $85.37 ; \mathrm{H}, 5.01 ; \mathrm{N}, 9.44$

2-(2-(Bis(1H-indol-3-yl)methyl)phenyl)-1H-benzimidazole (4i). (89\%), colorless crystals, $m p$ 179-181 ${ }^{\circ} \mathrm{C} ; \mathrm{IR}(\mathrm{KBr})$ $3392,3051,2955,2914,2850,1652,1463,1248,1209,804,739 \mathrm{~cm}^{-1}$; ${ }^{1} \mathrm{H}$ NMR (300 MHz, DMSO-d $d_{6}$, ref. DMSO$\left.d_{6}\right) \delta 6.09(\mathrm{~s}, 1 \mathrm{H}), 6.87(\mathrm{t}, J 6 \mathrm{~Hz}, 4 \mathrm{H}), 6.97-7.05(\mathrm{~m}, 5 \mathrm{H}), 7.29-7.48(\mathrm{~m}, 5 \mathrm{H}), 7.71(\mathrm{~s}, 2 \mathrm{H}), 7.93(\mathrm{~s}, 2 \mathrm{H}), 10.51(\mathrm{~s}, 1 \mathrm{H}$, $\mathrm{NH}), 11.27(\mathrm{~s}, 1 \mathrm{H}, \mathrm{NH}), 11.60(\mathrm{~s}, 1 \mathrm{H}, \mathrm{NH}) \mathrm{ppm} ;{ }^{13} \mathrm{C} \mathrm{NMR}\left(75 \mathrm{MHz}, \mathrm{DMSO}-d_{6}\right.$, ref. DMSO-d6) $\delta 41.2,110.6,112.2$, $115.4,118.7,119.9,120.4,121.4,123.1,127.4,128.5,129.6,130.4,136.1,136.8,138.4,141.5,153.4$ ppm; $\mathrm{MS}(\mathrm{EI}) \mathrm{m} / \mathrm{z}(\%) 438\left(\mathrm{M}^{+}, 1\right)$. FAB+ Calc. 438.1844, Found 438.1835.

2-(2-(Bis(1-methyl-1H-indol-3-yl)methyl)phenyl)-1H-benzimidazole (4j). (87\%), colorless crystals, $m p$ 148-151 ${ }^{\circ} \mathrm{C}$; IR (KBr) 3052, 2932, 2880, 1697, 1468, 1424, 1370, 1328, 790, $741 \mathrm{~cm}^{-1},{ }^{1} \mathrm{H}$ NMR (300 MHz, DMSO-d $d_{6}$, ref. DMSO- $\left.d_{6}\right) \delta 3.36(\mathrm{~s}, 6 \mathrm{H}), 6.90(\mathrm{~s}, 1 \mathrm{H}), 6.98-7.13(\mathrm{t}, J 6 \mathrm{~Hz}, 3 \mathrm{H}), 7.29-7.34(\mathrm{t}, J 6 \mathrm{~Hz}, 2 \mathrm{H}), 7.40-7.44(\mathrm{t}, J 3 \mathrm{~Hz}, 2 \mathrm{H})$, 7.46-7.56 (m, 6H), $7.87(\mathrm{~d}, J 6 \mathrm{~Hz}, 3 \mathrm{H}), 11.53(\mathrm{~s}, 1 \mathrm{H}, \mathrm{NH}) \mathrm{ppm} ;{ }^{13} \mathrm{C} \mathrm{NMR}\left(75 \mathrm{MHz}, \mathrm{DMSO}-d_{6}\right.$, ref. DMSO-d $d_{6} \delta 33.2$, 41.4, 109.4, 112.3, 115.3, 118.4, 119.4, 120.4, 121.4, 123.1, 126.4, 127.5, 128.2, 129.3, 130.1, 136.2, 137.4, 138.2, 141.6, 153.1 ppm; MS(EI) m/z (\%) 466 (M+, 13). ESI+ MS[1] Calc. 467.22357, Found 467.22253.

2-(2-(Bis(2-methyl-1H-indol-3-yl)methyl)phenyl)-1H-benzimidazole (4k). (86\%), colorless crystals, mp 225-227 ${ }^{\circ} \mathrm{C}$; IR (KBr) 3436, 3385, 3357, 3225, 3051, 2921, 1627, 460, 1320, 927, 812, $739 \mathrm{~cm}^{-1}$; ${ }^{1} \mathrm{H}$ NMR (300 MHz, DMSO$d_{6}$, ref. DMSO- $\left.d_{6}\right) \delta 2.09(\mathrm{~s}, 6 \mathrm{H}), 5.75(\mathrm{~s}, 1 \mathrm{H}), 6.87-6.93(\mathrm{~m}, 4 \mathrm{H}), 7.13-7.24(\mathrm{~m}, 9 \mathrm{H}), 7.41-7.44(\mathrm{~m}, 3 \mathrm{H}), 10.65(\mathrm{~s}$, $2 \mathrm{H}, \mathrm{NH}), 10.79(\mathrm{~s}, 1 \mathrm{H}, \mathrm{NH}) \mathrm{ppm} ;{ }^{13} \mathrm{C}$ NMR $\left(75 \mathrm{MHz}\right.$, DMSO- $d_{6}$, ref. DMSO-d 6 ) $\delta 12.2,41.4,111.3,112.5,115.0$, $118.8,119.6,120.2,121.6,123.7,127.2,128.2,129.4,130.6,131.0,136.2,138.4,141.2,152.8$ ppm; $M S(F A B+)$ $m / z(\%) 467\left(\mathrm{M}^{+}+1,12\right)$. ESI+ MS[1] Calc. 467.22357, Found 467.22235.

2-(2-(Bis(2-phenyl-1H-indol-3-yl)methyl)phenyl)-1H-benzimidazole (4l). (89\%), colorless crystals, $\mathrm{mp} 127-129$ ${ }^{\circ} \mathrm{C}$; IR (KBr) 3327, 3059, 2975, 1745, 1604, 1486, 1450, 796, 764, $746 \mathrm{~cm}^{-1} ;{ }^{1} \mathrm{H}$ NMR (300 MHz, DMSO- $d_{6}$, ref. DMSO- $\left.d_{6}\right) \delta 4.43(\mathrm{~s}, 1 \mathrm{H}, \mathrm{NH}), 5.85(\mathrm{~s}, 1 \mathrm{H}), 6.39(\mathrm{t}, J 3 \mathrm{~Hz}, 2 \mathrm{H}), 6.52(\mathrm{t}, J 3 \mathrm{~Hz}, 2 \mathrm{H}), 6.70-6.80(\mathrm{~m}, 5 \mathrm{H}), 6.84-6.92(\mathrm{~m}$, $10 \mathrm{H}), 7.02(\mathrm{~d}, J 6 \mathrm{~Hz}, 2 \mathrm{H}), 7.19-7.21(\mathrm{~m}, 5 \mathrm{H}), 10.82(\mathrm{~s}, 1 \mathrm{H}, \mathrm{NH}), 10.85$ (d, 1H', NH) ppm; ${ }^{13} \mathrm{C} \mathrm{NMR}(75 \mathrm{MHz}$, DMSO$d_{6}$, ref. DMSO- $\left.d_{6}\right) \delta 41.6,108.8,111.1,115.3,118.9,119.6,120.3,121.1,123.3,127.4,127.7,128.2,128.8$, 129.3, 129.6, 130.7, 133.0, 136.0, 136.8, 138.2, 141.6, 153.5 ppm; MS(FAB+) m/z (\%) $591\left(M^{+}+1,1\right)$. Elemental analysis: Calc. C, 85.40; H, 5.12; N, 9.48, Found. C, 85.40; H, 4.99; N, 9.37. 


\section{Acknowledgements}

José G. Penieres-Carrillo and Ricardo A. Luna-Mora acknowledge to DGAPA-UNAM the financial support to PAPIIT IN218312 and FES Cuautitlán PIAPIC14 projects.

\section{Supplementary Material}

Structure of synthesized compounds and IR, MS, ${ }^{1} \mathrm{H}$ NMR, ${ }^{13} \mathrm{C}$ NMR Spectra

\section{References}

1. Grose, K. R.; Bjeldanes, R.F. Chem. Res. Toxicol. 1992, 5, 188-193. http://dx.doi.org/10.1021/tx00026a007

2. Chen, I.; McDougal, A.; Wang, F.; Safe, S. Carcinogenesis 1998, 19, 1631-1639. http://dx.doi.org/10.1093/carcin/19.9.1631

3. Wattenberg, L. W.; Loub, W.D. Cancer Res. 1978, 38:1410-1413.

4. Auborn, K. J. Antivir. Ther. 2002, 7, 1-9.

5. Wiatrak, B. J. Curr. Opin. Otolaryngol. Head Neck Surg. 2003, 11, 433-441.

6. Navarrete-Vázquez, G.; Yépez, L.; Hernández-Campos, A.; Tapia, A.; Hernández-Luis, F.; Cedillo, R.; González, J.; Martínez-Fernández, A.; Martínez-Grueiro, M.; Castillo, R. Bioorg. Med. Chem. 2003, 11, 46154622.

http://dx.doi.org/10.1016/S0968-0896(03)00497-8

7. Özden, S.; Atabey, D.; Yıldız, S.; Göker, H. Bioorg. Med. Chem. 2005, 13, 1587-1597. http://dx.doi.org/10.1016/j.bmc.2004.12.025

8. Andrzejewska, M.; Yépez-Mulia, L.; Cedillo-Rivera, R.; Tapia, A.; Vilpo, L.; Vilpo, J.; Kazimierczuk, Z. Eur. J. Med. Chem. 2002, 37, 973-978.

http://dx.doi.org/10.1016/S0223-5234(02)01421-6

9. Terzioglu, N.; van Rijn, R. M.; Bakker, R. A.; De Esch, I. J. P.; Leurs, R. Bioorg. Med. Chem. Lett. 2004, 14, 5251-5256. http://dx.doi.org/10.1016/j.bmcl.2004.08.035

10. Remers, W. A.; Houlihan, W. J. (Ed.), Heterocyclic Compounds, Interscience Publishers, N.Y, 1972; p 1.

11. Chatterjee, A.; Manna, S.; Benerji, J.; Pascard, C.; Prangé, T.; Shoolery, J. N. J. Chem. Soc., Perkin Trans. I 1980, 553-555. http://dx.doi.org/10.1039/P19800000553

12. Babu, G.; Sridhar, N.; Petrumal, P. T. Synth. Commun. 2000, 30, 1609-1614. http://dx.doi.org/10.1080/00397910008087197

13. Chen, D.; Yu, L.; Wang, P. G. Tetrahedron Lett. 1996, 37, 4467-4470. http://dx.doi.org/10.1016/0040-4039(96)00958-6

14. Reddy, A. V.; Ravinder, K.; Reddy, V. L. N.; Goud, T. V.; Ravikanth, V.; Venkateswarlu, Y. Synth. Commun. 2003, 33. 3687-3694.

http://dx.doi.org/10.1081/SCC-120025177 
15. Navarrete-Vázquez, G.; Cedillo, R.; Hernández-Campos, A.; Yépez, L.; Hernández-Luis, F.; Valdez, J.; Morales, R.; Cortés, R.; Hernández, M.; Castillo, R. Bioorg. Med. Chem. Lett. 2001, 11, 187-190. http://dx.doi.org/10.1016/S0960-894X(00)00619-3

16. Ben-Alloum, A.; Bakkas, S.; Soufiaoui, M. Tetrahedron Lett. 1998, 39, 4481-4484. http://dx.doi.org/10.1016/S0040-4039(98)00868-5

17. Göker, H.; Ku, C.; Boykin, D. W.; Yildiz, S.; Altanar, N. Bioorg. Med. Chem. 2002, 10, 2589-2596. http://dx.doi.org/10.1016/S0968-0896(02)00103-7

18. Hermkens, P. H. H.; Ottenheijm, H. C. J.; Rees, D. C. Tetrahedron 1997, 53, 5643-5678. http://dx.doi.org/10.1016/S0040-4020(97)00279-2

19. Kappe, C. O. Angew. Chem. Int. Ed. 2004, 43, 6250-6284. http://dx.doi.org/10.1002/anie.200400655

20. Kappe, C. O. Chem. Soc. Rev., 2008, 37, 1127-1139 http://dx.doi.org/10.1039/b803001b

21. de la Hoz, A.; Díaz-Ortis, A.; Moreno, A.; Langa, F. Eur. J. Org. Chem. 2000, 3659-3673. http://dx.doi.org/10.1002/1099-0690(200011)2000:22<3659::AID-EJOC3659>3.0.CO;2-0

22. Dallinger, D.; Kappe, C. O. Chem. Rev. 2007, 107, 2563-2591. http://dx.doi.org/10.1021/cr0509410

23. Pore, D. M.; Desai, U. V.; Thopate, T. S.; Wadgaonkar, P. P. Arkivoc 2006, (xii), 75-80. http://dx.doi.org/10.3998/ark.5550190.0007.c09

24. Kamal, A.; Qureshi, A. A. Tetrahedron 1963, 19, 513-520. http://dx.doi.org/10.1016/S0040-4020(01)98540-0

25. Penieres-Carrillo, G.; García-Estrada, J. G.; Gutiérrez-Ramírez, J. L.; Álvarez-Toledano, C. Green Chem. 2003, 5, 337-339. http://dx.doi.org/10.1039/B211011C

26. Li, J.-T.; Dai, H.-G.; Xu, W.-Z.; Li, T.-S. Ultrason. Sonochem. 2006, 13, 24-27. http://dx.doi.org/10.1016/j.ultsonch.2004.12.004

27. Xia, M.; Wang, S.-H.; Yuan, W.-B. Synth. Commun. 2004, 34, 3175-3182. http://dx.doi.org/10.1016/j.arabjc.2011.02.009

28. Ji, S.-J.; Zhou, M.-F.; Gu, D.-G.; Jiang, Z.-Q.; Loh, T.-P. Eur. J. Org. Chem. 2004, 1584-1587. http://dx.doi.org/10.1002/ejoc.200300719

29. Pal, C.; Dey, S.; Mahato, S. K.; Vinayagam, J.; Pradhan, P. K.; Giri, V. S.; Jaisankar, P.; Hossain, T.; Baruri, S.; Raya, D.; Biswas, S. M. Bioorg. Med. Chem. Lett. 2007, 17, 4924-4928. http://dx.doi.org/10.1016/i.bmcl.2007.06.025

30. Yadav, J. S.; Reddy, B. V. S.; Sunitha, S. Adv. Synth. Catal. 2003, 345, 349-352.

31. Ma, Z.-H.; Han, H.-B.; Zhou, Z.-B.; Nie, J. J. Mol. Cat. A: Chem. 2009, 311, 46-53. http://dx.doi.org/10.1016/j.molcata.2009.06.021

32. Maiti, A. K.; Bhattacharyya, P. J. Chem. Res. (S) 1997, 424-425. http://dx.doi.org/10.1039/A701355H

33. Chakrabarty, M.; Ghosh, N.; Basaka, R.; Harigaya, Y. Tetrahedron Lett. 2002, 43, 4075-4078. http://dx.doi.org/10.1016/S0040-4039(02)00682-2

34. Olyyaei, A.; Vaziri, M.; Razeghi, R.; Shams, B.; Bagheri, H. J. Serb. Chem. Soc. 2013, 78, 463-468. http://dx.doi.org/10.2298/JSC1205060760

35. Feng, X.-L.; Guan, C.-J.; Zhao, C.-X. Synth. Commun. 2004, 34, 487-492. http://dx.doi.org/10.1081/SCC-120027288 
36. Lin, Z. H.; Guan, C. J.; Feng, X. L.; Zhao, C. X. J. Mol. Cat. A: Chem. 2006, 247, 19-26.

http://dx.doi.org/10.1016/j.molcata.2005.11.008

37. Azizi, N.; Manocheri, Z. Res. Chem. Intermed. 2012, 38, 1495-1500.

http://dx.doi.org/10.1007/s11164-011-0479-4

38. Magesh, C. J.; Nagarajan, R.; Karthik, M.; Perumal, P. T. Appl. Catal., A 2004, 266, 1-10.

http://dx.doi.org/10.1016/j.apcata.2004.01.024

39. Karthik, M.; Tripathi, A. K.; Gupta, N. M.; Palanichamy, M.; Murugesan, V. Catal. Commun. 2004, 5, 371375.

http://dx.doi.org/10.1016/j.catcom.2004.04.007

40. Naidu, K. R. M.; Khalivulla, S. I.; Kumar, P. C. R.; Lasekan, O. Org. Commun. 2012, 5, 150-159.

41. Zolfigol, M.A.; Salehi, P.; Shri, M.; Tanbakouchian, Z. Catal. Commun. 2007, 8, 173-178. http://dx.doi.org/10.1016/j.catcom.2006.06.012

42. Singh, K.; Sharma, S.; Sharma, A. J. Mol. Cat. A: Chem. 2011, 347, 34-37.

http://dx.doi.org/10.1016/j.molcata.2011.07.007

43. Gottlieb, H.E.; Kotlyar, V.; Nudelman, A. J. Org. Chem. 1997, 62, 7512-7515.

http://pubs.acs.org/doi/abs/10.1021/jo971176v 